\title{
Revista Colombiana de

\section{Capítulo 11. Utilidad del mapeo tridimensional en la ablación de la taquicardia ventricular isquémica}

\author{
Chapter 11. Usefulness of three-dimensional mapping \\ in ischemic ventricular tachycardia ablation
}

\author{
Juan C. Díaz ${ }^{\mathrm{a}}$, Julián M. Aristizábal ${ }^{\mathrm{a}}$, William Uribe ${ }^{\mathrm{a}, *}$, Guillermo Mora ${ }^{\mathrm{b}, \mathrm{c}, \mathrm{d}}$, \\ Jorge E. Velásquez ${ }^{\mathrm{a}}$ y Mauricio Duque ${ }^{\mathrm{a}}$
}

a Servicio CES Cardiología, Medellín, Colombia

b Universidad Nacional de Colombia, Bogotá, Colombia

' Fundación Santafé de Bogotá, Bogotá, Colombia

d Hospital Universitario Clínica San Rafael, Bogotá, Colombia

Recibido el 15 de noviembre de 2015; aceptado el 18 de enero de 2016

\section{Introducción}

La enfermedad coronaria es, según cifras publicadas por el Ministerio de Salud y Protección Social, la principal causa de muerte en Colombia, especialmente en personas mayores de 55 años $^{1}$. En el año 2011 se estimó que murieron en total 29.000 personas (un promedio de 80 personas al día) a causa de infartos agudos de miocardio en Colombia, 55\% de ellos varones ${ }^{2}$. Con los avances en el manejo médico, cada vez son más los pacientes que sobreviven a un evento coronario; a pesar de ello y debido a una prevalencia en aumento de la enfermedad coronaria, el número total de muertes atribuibles a ello ha mostrado un ascenso en las últimas tres décadas en el país ${ }^{3,4}$. Muchos sobrevivientes tendrán secuelas que variarán de acuerdo con la extensión del daño producido por la isquemia y que incluyen angina crónica, falla cardiaca y arritmias ventriculares (taquicardia ventricular y fibrilación ventricular). La taquicardia ventricular (definida como la presencia de tres o más latidos consecutivos que se originan por debajo de la división del haz de His) es la principal causa de muerte súbita en pacientes con cardiopatía isquémica y se asocia con aumento en la mortalidad y disminución en la calidad de vida en pacientes con desfibriladores. Esto ha llevado a que en la actualidad se sugiera la ablación de manera temprana en el manejo de estas arritmias ${ }^{5}$. Debido a las características de la arritmia y a las dificultades técnicas asociadas con su ablación, la mayoría de las nuevas tecnologías son adoptadas con base en estudios pequeños no aleatorizados, lo cual tiene un impacto significativo en cuanto al nivel de evidencia que respalda muchas de las intervenciones. En esta sección se revisará la utilidad de los sistemas de mapeo tridimensional en la ablación de taquicardia ventricular isquémica. 


\section{Morfología de la taquicardia y su relación con el mecanismo de la arritmia}

La morfología de la taquicardia ventricular puede dar pistas importantes sobre el origen de la arritmia. La taquicardia ventricular monomórfica (aquella que tiene una morfología que permanece constante durante varios latidos) es producto de una secuencia de activación ventricular que permanece constante. Dicha arritmia se asocia con mecanismos que involucran un aumento del automatismo o circuitos de reentrada, por lo cual es la arritmia más encontrada en pacientes con cardiopatía isquémica. Cabe resaltar que un mismo paciente puede tener más de un circuito de reentrada (o un foco automático tener más de un sitio de salida), por lo que es posible tener múltiples morfologías de taquicardia ventricular monomórfica en un mismo paciente; esto es importante al momento de realizar ablación de la arritmia ya que en lo posible se debe buscar la terminación de todas las taquicardias ventriculares. Por su parte, la taquicardia ventricular polimórfica (aquella cuya morfología cambia de manera continua durante la taquicardia, sin conservar una misma morfología por más de cinco latidos) se asocia por lo general a actividad desencadenada, y por tanto a isquemia aguda (o desórdenes genéticos, electrolíticos o fármacos que alteran la duración del intervalo QT) más que con cicatrices existentes. Por último, la taquicardia ventricular bidireccional (aquella cuyo eje en el plano frontal cambia latido a latido) se relaciona con intoxicación digital y taquicardia ventricular catecolaminérgica. De lo anterior se puede inferir que la mayor parte de las taquicardias ventriculares isquémicas que se manejan en la sala de electrofisiología, serán taquicardias ventriculares monomórficas asociadas a cicatrices por eventos isquémicos antiguos.

\section{La cicatriz isquémica y su papel en la génesis de las arritmias ventriculares}

Durante un evento isquémico agudo se produce la muerte de miocitos, que posteriormente serán reemplazados por tejido fibrótico (el cual no permite la conducción de impulsos eléctricos) generando áreas de bloqueo en la conducción del impulso. Las lesiones isquémicas no producen daño uniforme; afectan más al endocardio que al epicardio (debido a las características de la circulación coronaria, donde el endocardio es la zona más vulnerable a la isquemia) y las zonas más alejadas de los vasos sanguíneos. Es así como la cicatriz no es una zona uniformemente necrótica, sino más bien una en la cual se intercalan áreas de fibrosis con miocitos sanos que forman canales dentro de las cicatrices. Esta mezcla de tejido fibrótico con tejido viable, genera retardos en la conducción, bloqueos y cambios en la dirección de conducción del impulso (conducción anisotrópica), lo que a su vez crea circuitos de reentrada (fig. 1). A esto se suman alteraciones en los canales iónicos y en las uniones gap que enlentecen aún más la conducción, incluso entre miocitos viables ${ }^{6}$. Por lo general, los canales que sostienen taquicardias ventriculares son más largos, tienen propiedad de conducción más lenta y están ubicados en el interior de las cicatrices más densas, que los canales que no sostienen taquicardias ventriculares ${ }^{7}$. Estos circuitos son muy estables en el tiempo, por lo que la tasa de recurrencias puede superar el $40 \%$ en los primeros

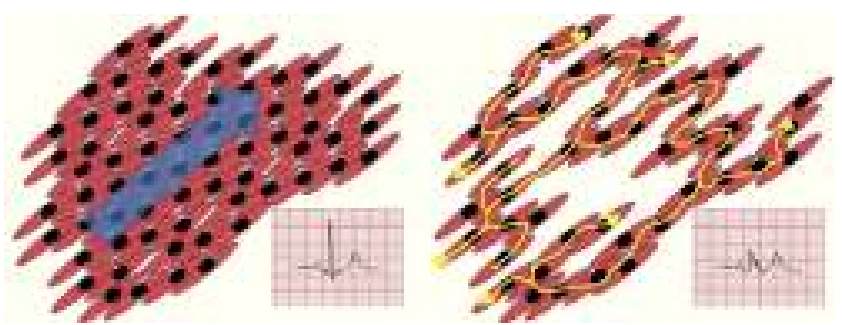

Figura 1 En condiciones normales, la conducción del miocardio sano se produce en el sentido de las fibras musculares, ocasionando una despolarización casi simultánea de todas las células (izquierda), lo cual puede observarse en el electrocardiograma de superficie como un QRS angosto. En las áreas cicatriciales (derecha), hay zonas de tejido fibrótico intercaladas con miocitos viables. La conducción no es siempre en sentido longitudinal, característica que produce una conducción tres a cuatro veces más lenta. Se forman zonas de conducción lenta y los distintos impulsos eléctricos no llevan la misma dirección de despolarización, lo cual se traduce en la formación de circuitos de reentrada. Esta despolarización anisotrópica puede observarse como fragmentación y disminución del voltaje del QRS en el electrocardiograma de superficie.

dos años después del evento arrítmico inicial ${ }^{8}$. De manera interesante, los mecanismos de remodelación que siguen a un evento isquémico del miocardio parecen favorecer de modo significativo la génesis de las arritmias. Hay estudios que han demostrado la aparición de las arritmias ventriculares, varios años después del evento agudo?.

En la creación del circuito participan, entonces, bloqueos funcionales y anatómicos, que generan una zona protegida de conducción lenta (istmo crítico) con un sitio de salida que conecta con el miocardio ventricular (produciendo la despolarización) y un sitio de entrada al istmo (fig. 2$)^{10}$. Estos istmos por lo general están ubicados en forma paralela al plano mitral en taquicardias perimitrales, y perpendicular al plano mitral en taquicardias de otra orientación, lo cual debe tenerse en cuenta al momento de hacer la ablación de la arrit$\mathrm{mia}^{11}$. Estas zonas de conducción lenta no son visibles en el electrocardiograma de superficie; no obstante, al ubicar un catéter de registro sobre ellas se pueden observar potenciales fraccionados (señales con múltiples componentes de alta frecuencia, baja amplitud y larga duración, indicativos de conducción anisotrópica y por ende lenta) o potenciales desdoblados (split: potenciales separados por un período isoeléctrico de al menos 30 a $50 \mathrm{~ms}$ ).

\section{Evaluación del paciente con cardiopatía isquémica y taquicardia ventricular}

La evaluación del paciente busca identificar condiciones que podrían poner en riesgo su vida durante la ablación o que dificultarían el procedimiento como tal. Es importante llevar al paciente compensado desde el punto de vista hemodinámico, prestando atención especial a la sobrecarga hídrica ya que los procedimientos de ablación de taquicardia ventricular son largos, lo cual, sumado a la descompensación propia de la inducción de arritmias (por la pérdida de la sincronía aurículo-ventricular) y al uso de catéteres de ablación con irrigación 


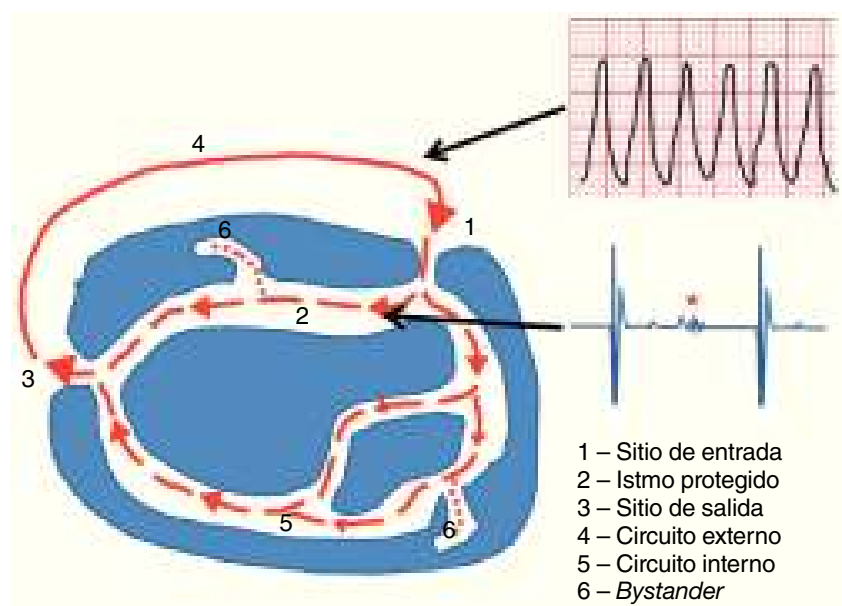

Figura 2 Esquema en el que se representan los componentes de un circuito de reentrada. Durante una taquicardia ventricular, el circuito se sostiene utilizando un istmo protegido; la presencia de circuitos internos adyacentes no hace parte del circuito de esa taquicardia. Sin embargo, estos circuitos pueden a su vez formar taquicardias con una morfología similar pero con una longitud de ciclo distinta (la cual dependerá de la longitud del circuito en cuestión y de la velocidad de conducción a través de él). Al salir, el impulso del istmo protegido (es decir, recorriendo el asa externa) produce la despolarización del ventrículo, lo cual es visible en el electrocardiograma de superficie. El paso del estímulo por el istmo protegido no es visible en el electrocardiograma de superficie; no obstante, al ubicar un catéter sobre esta zona se verá durante la taquicardia un potencial medio diastólico (estrella roja).

externa, puede llevar a sobrecarga hídrica y edema pulmonar. Vale la pena resaltar que la presencia de tormenta eléctrica no es una contraindicación para la ablación, y que, por el contrario, estos pacientes se benefician notablemente de este procedimiento con una tasa de éxito temprano de hasta el $89 \%$ (pese a que con frecuencia requerirán soporte circulatorio durante la taquicardia ventricular $)^{12}$. Adicionalmente, es pertinente contar con una evaluación ecocardiográfica, no solo para determinar la función ventricular sino para determinar si existen alteraciones estructurales (aneurismas, trastornos valvulares, trombos) que puedan complicar el procedimiento.

Como ya se estableció, la isquemia aguda es una causa poco frecuente de taquicardia ventricular monomórfica sostenida, ya que estas se deben por lo general a circuitos de reentrada asociados a cicatrices preexistentes ${ }^{13,14}$. No obstante, conviene descartar la presencia de isquemia residual, ya que esto modifica el pronóstico de la enfermedad y disminuye la tasa de recurrencias de la arritmia al reducir los disparadores. Esto puede hacerse mediante pruebas no invasivas (perfusión miocárdica o ecocardiografía de estrés con ejercicio o con fármacos) o coronariografía, de acuerdo con la sintomatología del paciente.

\section{Ablación de la taquicardia ventricular isquémica con catéter}

Debido al carácter crónico, y en la mayoría de los casos progresivo, de la enfermedad coronaria, la ablación con catéter no es una medida que permita la curación definitiva de las arritmias ventriculares ni la prevención absoluta de un episodio de muerte súbita. En este sentido, debe considerarse el uso de cardiodesfibriladores como estrategia de prevención primaria o secundaria en pacientes apropiados, y brindar terapia médica óptima con el fin de disminuir el riesgo de nuevos eventos isquémicos o arrítmicos. La ablación con catéter es pues una medida coadyuvante para el manejo de las arritmias, ya sea en el caso de las taquicardias ventriculares que recurren pese al manejo médico o en el caso de los pacientes que se presentan con tormenta eléctrica, e incluso tiene valor como medida preventiva para disminuir el riesgo de arritmias ventriculares y descargas apropiadas en los portadores de cardiodesfibriladores (CDI) ${ }^{15}$.

Existen dos estrategias (en muchos casos convergentes) para el manejo de la taquicardia ventricular isquémica: la ablación de las arritmias inducibles y la modificación de sustrato. En la primera estrategia se busca identificar el origen de la arritmia y realizar ablaciones puntuales que lleven a su terminación. En el segundo caso se pretende modificar la cicatriz isquémica de tal forma que no permita la perpetuación de la arritmia.

\section{Ubicación electrocardiográfica de la arritmia}

Debido a la presencia de zonas cicatriciales de mayor o menor extensión, la ubicación electrocardiográfica de la arritmia es menos precisa que la ubicación de taquicardias ventriculares focales en corazones sanos, aun con el uso de modelos computarizados ${ }^{16}$. No obstante, las características electrocardiográficas de la arritmia permiten realizar una aproximación hacia la zona comprometida, facilitando los esfuerzos iniciales de mapeo. En primer lugar, la mayoría de las taquicardias ventriculares isquémicas tendrá un origen a nivel del ventrículo izquierdo, por lo que se espera una morfología de bloqueo de rama derecha, mientras que las que presentan morfología de bloqueo de rama izquierda por lo general tendrán un sitio de salida en la región septal. En el plano frontal, el eje del QRS ayuda a establecer un origen en la parte baja septal (eje izquierdo, despolarización superior) de las que se originan en la pared posterior o lateral del ventrículo izquierdo (eje derecho, despolarización en dirección inferior). En infartos de la pared inferior, la despolarización del ventrículo se hace desde posterior hacia anterior, por lo que habitualmente se observa una onda R prominente de V2 a $\mathrm{V}^{17}$. Al producir mayor destrucción de masa miocárdica, los infartos de pared anterior hacen que la ubicación electrocardiográfica sea aún menos confiable.

\section{Origen real vs. aparente}

Teniendo en cuenta el mecanismo de la taquicardia ventricular isquémica, es fácil comprender por qué puede existir una distancia significativa entre el origen aparente (dado por el sitio de salida del istmo protegido) y el origen real de la arritmia (istmo protegido). Esto es de particular relevancia al momento de realizar técnicas de mapeo de activación (incluyendo el mapeo efectuado con sistemas tridimensionales; ver más adelante), ya que la activación más precoz se encontrará en el sitio de salida pero la ablación en esta zona no terminará la arritmia. 


\section{Mecanismo de la arritmia: la importancia de las maniobras de encarrilamiento}

Las maniobras de encarrilamiento permiten diferenciar un foco automático de un foco por reentrada, siendo este el mecanismo más común en las taquicardias ventriculares isquémicas. Para realizar el encarrilamiento, las taquicardias deben ser bien toleradas desde el punto de vista hemodinámico, o en su defecto, contar con soporte hemodinámico como el uso del Impella (soporte de asistencia ventricular percutáneo). La capacidad de realizar de manera exitosa el encarrilamiento hace el diagnóstico de un mecanismo de reentrada. Estas maniobras son complementos importantes al mapeo tridimensional.

\section{Uso de tecnologías de mapeo tridimensional en la ablación de taquicardia ventricular isquémica}

En la actualidad, los sistemas de mapeo tridimensional son pieza clave en el manejo de la taquicardia ventricular isquémica, dada la capacidad de estos sistemas de facilitar la identificación de zonas de interés durante la ablación. Actualmente, en Colombia se cuenta con dos sistemas de mapeo tridimensional: el EnSite ${ }^{\circledR}$ (St. Jude Medical, St Paul, Minnesota, Estados Unidos) y el CARTO ${ }^{\circledR}$ (Biosense Webster, Diamond Bar, California, Estados Unidos).

Independiente del que se utilice, los sistemas de navegación tridimensional desempeñan un papel fundamental en la ablación de la taquicardia ventricular isquémica. Los beneficios de utilizar estas tecnologías incluyen:

1. Disminución de la radiación iónica al paciente, al operador y al personal de la sala. Al realizar una reconstrucción tridimensional, el movimiento de los catéteres puede ser seguido sin la necesidad de fluoroscopia ${ }^{18-20}$. Aunque no existen estudios específicamente realizados en pacientes con taquicardia ventricular, la extrapolación de los resultados de la ablación de otros tipos de arritmias (particularmente supraventriculares) permite predecir el impacto de los sistemas de mapeo en la reducción de tiempo de fluoroscopia.

2. Mayor precisión al momento de identificar la posición del catéter dentro de la cámara cardiaca.

3. Posibilidad de realizar mapas de voltaje, permitiendo identificar de esta manera las zonas con cicatriz eléctrica, lo que a su vez ayuda a enfocar los esfuerzos de mapeo y ablación en esta zona.

4. Marcación de zonas de interés por su potencial arrítmico. Esto es particularmente importante en las técnicas de modificación de sustrato, donde se realiza ablación buscando homogenización de la cicatriz con el fin de evitar la reaparición de arritmias.

Debido a estas ventajas sobre el mapeo fluoroscópico, los sistemas de mapeo tridimensional han tenido una gran acogida y actualmente son parte fundamental en los procedimientos de ablación de taquicardia ventricular isquémica. Por esta razón, no existen estudios aleatorizados que comparen el mapeo fluoroscópico vs. el no fluoroscópico en esta condición clínica con el fin de determinar las tasas de éxito, y hoy se considera que un estudio de estas características no sería ético ${ }^{21}$. Sin embargo, un estudio pequeño demostró mayor tasa de éxito y menor tiempo de fluoroscopia en la ablación de taquicardia ventricular del tracto de salida del ventrículo derecho al comparar un sistema de navegación tridimensional (en este caso, CARTO) contra el mapeo fluoroscópico ${ }^{22}$. Es de esperarse entonces que los resultados en la ablación de taquicardia ventricular isquémica, una arritmia mucho más compleja que la taquicardia ventricular del tracto de salida del ventrículo derecho, sean superiores al utilizar un sistema de mapeo tridimensional.

\section{Integración con imágenes diagnósticas}

La integración de las técnicas de mapeo tridimensional con imágenes diagnósticas (ecocardiografía intracardiaca, resonancia magnética, tomografía computarizada) permite no solo realizar un mapa muy bien estructurado del ventrículo izquierdo y las demás estructuras anatómicas, sino detectar zonas cicatriciales e incluirlas en los mapeos sin necesidad de tomar mediciones punto a punto, acelerando el proceso del mapeo de voltaje ${ }^{23}$.

\section{Técnicas de mapeo}

Durante el mapeo de la taquicardia ventricular isquémica, es posible realizar mapeos de activación, de potenciales fragmentados complejos y de voltaje o cicatrización (figs. 3-5).

\section{Mapeo de activación}

En el mapeo de activación se busca detectar sitios de activación precoz durante taquicardia (correspondiente al sitio de salida del istmo protegido) o la detección de señales sugestivas de canales e istmos (actividad diastólica durante taquicardia o potenciales tardíos durante sinusal). En este último, vale la pena resaltar que la presencia de potenciales tardíos y/o fraccionados refleja una zona de miocardio enfermo, con conducción lenta; sin embargo, esta zona no necesariamente estará en relación con el circuito de la taquicardia. Se sugiere el uso de electrogramas bipolares, ya que estos tienen una mejor relación entre ruido y señal y por ende dan un registro de mejor calidad. La relación de la primera señal de activación local (bien sea un potencial presistólico durante taquicardia ventricular o la señal local bipolar) con el QRS de superficie, permite ubicar la zona de mayor precocidad. En los sistemas de mapeo tridimensional, estas son representadas en un mapa de colores. Debe tenerse en cuenta que el mapa de activación permite únicamente la ubicación de la primera zona en activarse, por lo que durante una taquicardia ventricular asociada a un circuito de reentrada ésta estará ubicada en el punto de salida del istmo protegido, y como ya se estableció, la ablación en este punto no va a ser efectiva.

\section{Ubicación de potenciales fraccionados y medio-diastólicos}

Utilizando el mapeo tridimensional, es posible realizar una reconstrucción de la cavidad endocárdica buscando y marcan- 


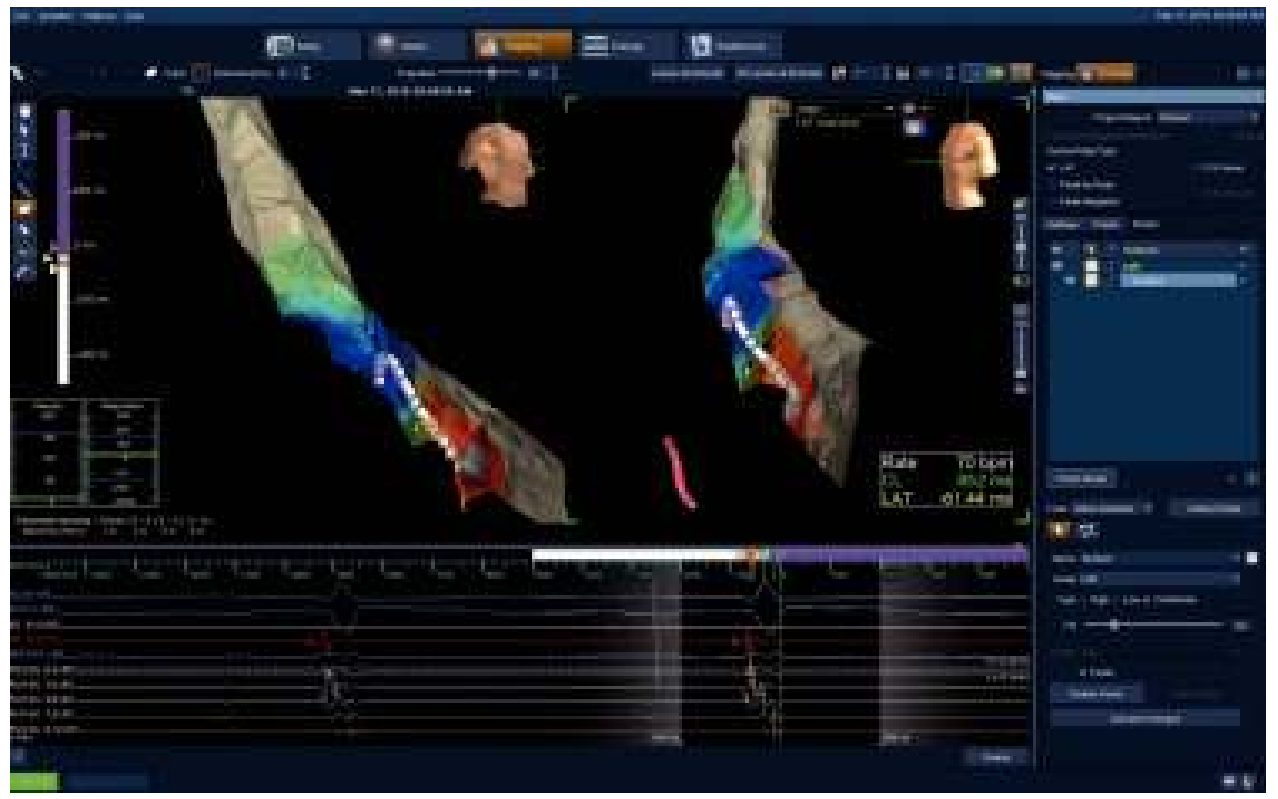

Figura 3 Inicio de una reconstrucción 3-D del ventrículo izquierdo y de un mapa de activación utilizando un catéter de mapeo decapolar de St. Jude Medica ${ }^{\circledR}$. Se puede observar a la izquierda la escala de colores con asignación de intervalos de tiempo en ms en donde el blanco y el rojo representarán las zonas de mayor precocidad y el azul y el púrpura, las zonas más tardías. Abajo se observa el electrocardiograma, el electrograma del ventrículo derecho y los electrogramas ventriculares del catéter de mapeo. Imagen del laboratorio de Electrofisiología del grupo CES Cardiología.

do las áreas en las cuales se encuentran potenciales fraccionados. De igual manera, durante taquicardia es factible identificar zonas de potenciales medio-diastólicos. Si bien estas señales son indicativas de zonas cicatriciales con conducción lenta (el sustrato básico para las taquicardias ventriculares isquémicas), estos no necesariamente están implicados en el circuito de la arritmia. En estos casos se evalúa mediante maniobras electrofisiológicas si hacen parte o no del circuito.

\section{Mapeo de voltaje}

Durante el mapeo de voltaje, el sistema de navegación tridimensional asigna un color a los voltajes encontrados durante la activación ventricular en ritmo sinusal. De esta manera se pueden delimitar las áreas de miocardio sano (voltaje mayor a 1,5 mV), la zona de transición (sitio de gran interés debido a su participación en los circuitos de reentrada, identificada

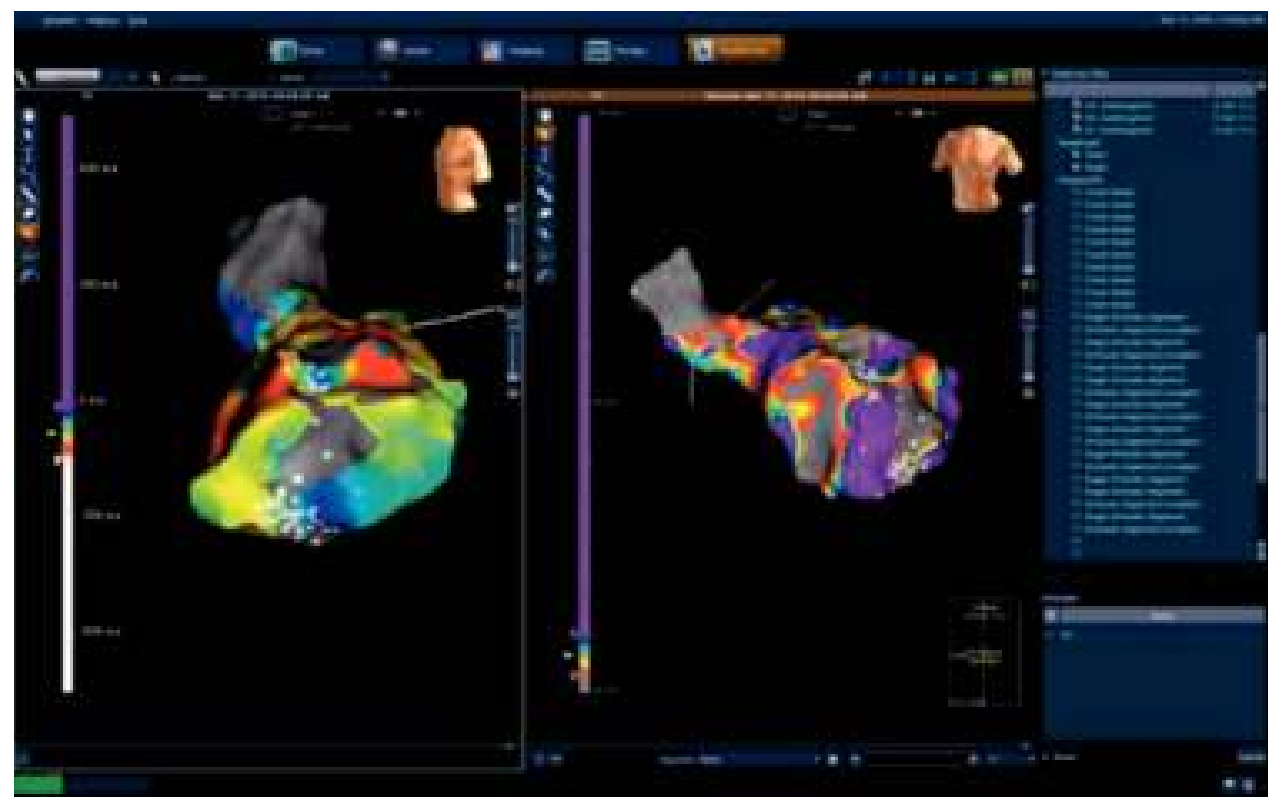

Figura 4 Mapa anatómico (reconstrucción geométrica) terminado del paciente anterior. A la izquierda se muestra el mapa de activación con escala de colores en ms y a la derecha se observa el mapa de voltaje o cicatriz en mV. Se señalan los sitios con potenciales diastólicos (DP) en ritmo sinusal. Imagen del laboratorio de Electrofisiología del grupo CES Cardiología. 


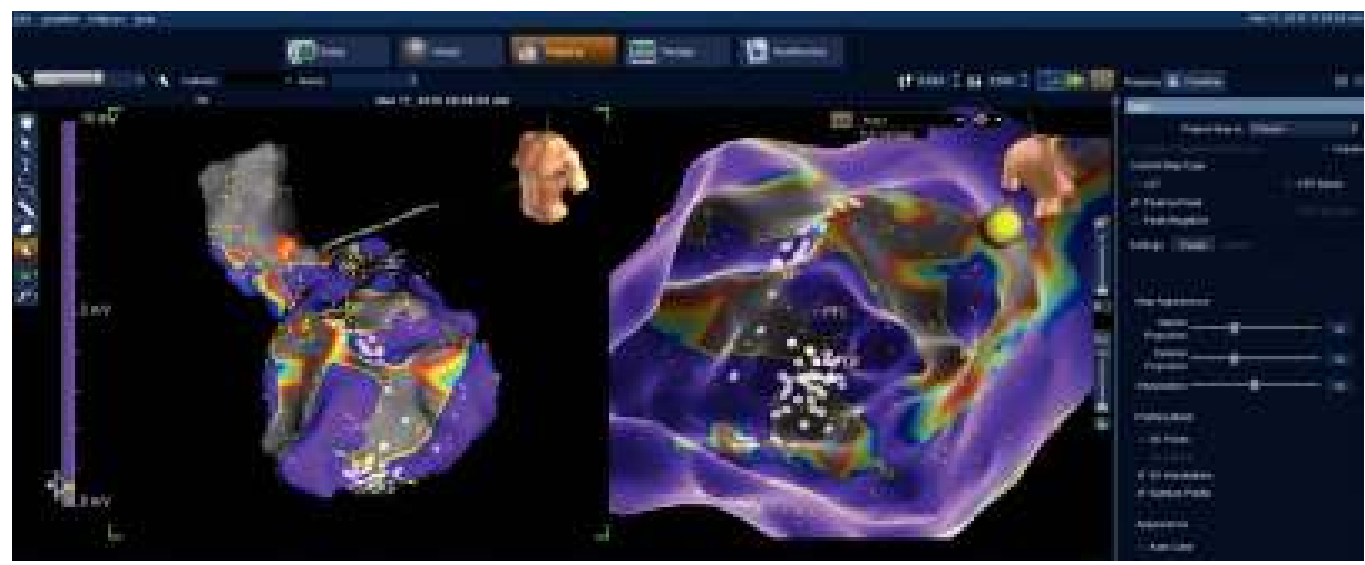

Figura 5 Figura izquierda: vista externa. Figura derecha: vista interna. Mapa de voltaje del paciente anterior, que muestra claramente las zonas de cicatrización con voltajes menores de $0,5 \mathrm{mV}$ en gris, zonas sanas en púrpura (> 1,5 mV) y zonas limítrofes o borde de cicatriz en colores rojo, amarillo y azul en diferentes tonos (entre 0,5 y 1,5 mV). El punto amarillo 3-D corresponde a la localización anatómica del haz de His. Los puntos blancos a los sitios de ablación en las zonas con canales, potenciales diastólicos y potenciales fraccionados. DP: potencial diastólico, PF: potencial fraccionado, PP: potencial precoz. Imagen del laboratorio de Electrofisiología del grupo CES Cardiología.

por un voltaje entre $0,5-1,5 \mathrm{mV}$ ) y la zona de cicatriz (voltaje $<0,5 \mathrm{mV})$. Es fundamental asegurar un excelente contacto entre el catéter y el tejido, ya que de lo contrario la pérdida de voltaje asociada con un contacto pobre será interpretada por el sistema de manera errónea como una zona cicatricial $^{24}$. Una vez delimitada la zona cicatricial, es posible detectar la presencia de canales mediante la estimulación con el catéter: los canales tienen por lo común un umbral de menos de $10 \mathrm{~mA}$. Adicionalmente, modificar la definición de cicatriz (disminuyendo el voltaje de $0,5 \mathrm{mV}$ a 0,3 o incluso $0,1 \mathrm{mV}$ ) puede ayudar a visualizar estos canales para su posterior ablación; no obstante, esta aproximación identifica una gran cantidad de "canales" que no tienen relación con las taquicardias clínicamente demostradas, encontrándose a distancias de hasta 2,4 cm de los istmos críticos de la arritmia. Esta aproximación, por sí sola, arroja una sensibilidad y especificidad del 46 y 33\% para la ubicación de canales en los que se ubique un istmo crítico. Pese a ello, la presencia de potenciales aislados tardíos mejora estas cifras a 78 y $85 \%$ de manera respectiva, haciéndolos atractivos para la ablación de sustrato, especialmente si se tiene en cuenta que estos canales que contienen istmos por lo general se asocian con taquicardias ventriculares rápidas ${ }^{25}$. La participación de los canales documentados de esta manera en el circuito de la taquicardia ventricular, se confirma mediante técnicas electrofisiológicas convencionales.

\section{Mapeo de no contacto}

En pacientes con taquicardias ventriculares inestables o de corta duración, el mapeo de no contacto (EnSite 3000, St. Jude Medical) puede ser una alternativa viable para obtener información con unos pocos latidos de taquicardia ${ }^{26-29}$. En esta técnica se utiliza un balón de $7,5 \mathrm{ml}$ con múltiples electrodos en su superficie el cual no entra en contacto con las paredes miocárdicas, y mediante una serie de algoritmos es capaz de calcular los potenciales eléctricos a una distancia máxima de $40 \mathrm{~mm}$ desde el centro del balón; puede realizar la proyección de hasta 3.000 señales unipolares en la superficie endocárdica. En cavidades muy dilatadas, se debe reposicionar el catéter con el fin de obtener información fidedigna. Utilizando el mapeo de no contacto es posible realizar un mapa del sustrato isquémico (mapeo de voltaje) con apenas unos segundos de ritmo sinusal; posteriormente se induce la taquicardia y en apenas unos pocos latidos se puede obtener hasta en el $90-99 \%$ de los pacientes información sobre el sitio de salida y en algunos casos (67-80\%) sobre la presencia de istmos de conducción lenta ${ }^{26,29}$.

Existen varias limitaciones respecto al uso del mapeo de no contacto en la ablación de taquicardia ventricular isquémica. En primer lugar, hasta el momento hay muy poca evidencia que avale su uso, ya que la inmensa mayoría de los estudios de ablación en taquicardia ventricular isquémica se realizan con mapeo de contacto. En segundo lugar, debido a las características del catéter balón, se requiere anticoagular al paciente manteniendo un ACT (tiempo de coagulación activado, por sus siglas en inglés) por encima de 350 segundos con el fin de evitar formación de trombo en la superficie del balón. Por último, puede ser difícil ubicar adecuadamente el catéter en la cavidad, ya sea por dificultades para avanzar el catéter hacia el interior del ventrículo izquierdo (tortuosidad de la aorta, aterosclerosis) o por la necesidad de reposicionar el catéter varias veces en corazones muy dilatados con el fin de obtener registros fidedignos.

\section{Ablación de sustrato}

La ablación de sustrato ha surgido como una técnica para la ablación de taquicardias ventriculares no inducibles o hemodinámicamente mal toleradas, basándose en la eliminación de canales y sitios de salida mediante la homogenización del sustrato. Este tipo de estrategias buscan además disminuir la tasa de recurrencia de las taquicardias ventriculares, la cual es bastante alta a pesar de utilizar como meta durante la ablación, la incapacidad de inducir arritmias ventriculares después de la aplicación de radiofrecuencia. 


\section{Lesiones lineares}

Descrita de manera reciente, la ablación linear busca eliminar los circuitos mediante la creación de lesiones lineares que interrumpen la zona limítrofe entre miocardio sano y miocardio anormal. Las lesiones se ubican a lo largo de la zona de voltaje anormal, llevando las líneas hacia miocardio sano y en lo posible buscando la continuidad valvular para ser usada como sitio de anclaje. En esta técnica se hace énfasis especial en las zonas donde la topoestimulación mostraba una morfología similar a la de la taquicardia ventricular. Esta técnica muestra una tasa de éxito aceptable (75\%) y se convirtió en una de las primeras tecnologías descritas para la modificación de sustrato en pacientes con taquicardia ventricular no sostenible o inestable ${ }^{30}$.

\section{Eliminación de actividad ventricular local anormal}

La actividad ventricular local anormal (LAVA, su sigla en inglés por local abnormal ventricular activities) son todas aquellas señales tardías y de alta frecuencia claramente distinguibles del electrograma ventricular y que pueden aparecer durante o después de este en ritmo sinusal o precediendo al electrograma ventricular durante taquicardia ventricu$\operatorname{lar}^{31}$. Como ya se estableció, los potenciales fraccionados pueden encontrarse a lo ancho de la cicatriz isquémica y por ende su presencia no implica relación con circuitos de reentrada. Por el contrario, los potenciales tardíos sí tienen una estrecha relación con circuitos de taquicardia y son el blanco de estrategias de modificación de sustrato ${ }^{25,32}$. Para su identificación, se requiere con frecuencia la estimulación desde distintas partes del ventrículo derecho e izquierdo con el fin de separar estos componentes del electrograma ventricular; se ha descrito su ablación tanto endocárdica como epicárdica (esta última cuando no se encuentran o no se eliminan los LAVA desde el endocardio) $)^{31}$.

La principal desventaja de esta técnica de ablación consiste en la gran cantidad de potenciales tardíos que no se correlacionan con la génesis de la arritmia y que simplemente son observadores (bystanders); estos parecen estar más comúnmente asociados a infarto de pared inferior y pared lateral $^{33,34}$ Su ablación, por tanto, aumenta el tiempo y la complejidad del procedimiento sin incrementar la tasa de éxito. Por último, es importante tener en cuenta que al eliminar el LAVA más precoz, se pueden eliminar varios LAVA (ya que la señal más temprana representa el sitio de entrada a un canal), con lo cual se evita realizar lesiones adicionales.

\section{Eliminación de canales y homogenización de la cicatriz}

De manera reciente, se ha propuesto la eliminación de canales intracicatriciales (scar dechanneling) como una estrategia de ablación antes de iniciar las técnicas de mapeo y ablación convencional ${ }^{35}$. Utilizando un sistema de mapeo tridimensional, se identifica la zona cicatricial (utilizando un voltaje < $0,5 \mathrm{mV}$ para definir cicatriz $\mathrm{y}>1,5 \mathrm{mV}$ para definir tejido sano) y en la zona borde o límite, entre la cicatriz eléctrica y el tejido sano, se marcan las áreas con potenciales tardíos de alta frecuencia. Se evita la zona de miocardio sano durante el mapeo, y aquellos con los potenciales anormales más tempranos son sometidos a ablación (asumiendo que estas áreas con potenciales tardíos cercanos al electrograma de ventrículo corresponden al sitio de entrada de los canales). La eliminación de los canales se logra en el $84 \%$ de los casos, encontrando en promedio 8 canales por paciente, los cuales pueden requerir de hasta 18 aplicaciones de radiofrecuencia para su eliminación ${ }^{35}$. Con esta estrategia se busca limitar el número de aplicaciones de radiofrecuencia (en comparación con las técnicas de eliminación de LAVA), y al iniciar con ésta se logra la meta de no inducibilidad en el 54,5\% de los pacientes. Al hacer posteriormente ablación de las taquicardias ventriculares inducibles, se logra la meta de no inducibilidad en el $78 \%$ de los casos. Adicionalmente, en quienes se logra la no inducibilidad con la estrategia de eliminación de canales únicamente, se observa una menor tasa de recurrencia de taquicardia ventricular.

\section{Metas u objetivos (targets) de la ablación}

Existen varias metas para considerar como exitosa una ablación de taquicardia ventricular isquémica:

- Incapacidad para inducir taquicardia ventricular. Es el desenlace tradicionalmente aceptado, a pesar de que se logra en tan solo el $64 \%$ de los pacientes y que no hay evidencia contundente que demuestre una menor recurrencia de la arritmia ${ }^{5,36}$. Al respecto, es importante tener en cuenta que la inducción de una taquicardia ventricular sigue un comportamiento probabilístico, mas no determinístico. Adicionalmente, la técnica de estimulación influye de manera significativa en la capacidad de inducción: es mayor al realizar inducción con estimulación ventricular izquierda y al usar estimulación desde varios puntos del ventrículo derecho ${ }^{37-39}$.

- Demostración del bloqueo de la conducción cuando se realiza una línea de ablación. Esta demostración se puede basar en la realización de un mapa de activación utilizando un sistema tridimensional. Esta meta no ha sido evaluada en estudios prospectivos.

- Eliminación de todos los LAVA presentes en la cicatriz miocárdica ${ }^{31,40,41}$. Con la tecnología actual esto es posible en aproximadamente un $70 \%$ de los $\operatorname{casos}^{31}$. Este desenlace ha sido utilizado en estrategias de modificación de sustrato.

- Incapacidad para lograr la captura con salidas elevadas (hasta $20 \mathrm{~mA}$ con una anchura de pulso de $10 \mathrm{~ms}$ ) ${ }^{41}$. Este desenlace ha sido utilizado en estrategias de modificación de sustrato en conjunto con la eliminación de LAVA como estrategia de modificación del sustrato.

\section{Desventajas de los sistemas de mapeo tridimensional}

La principal debilidad de los sistemas de mapeo tridimensional tiene que ver con la precisión con la cual se representa el catéter dentro de la cavidad ventricular virtual. La respiración y los movimientos cardiacos y del catéter pueden disminuir esta precisión. En particular, los movimientos de la referencia eléctrica pueden tener un impacto marcado en los 
mapas, en ocasiones llegando a invalidarlos (lo que obliga a la creación de un nuevo mapa). Adicionalmente, el mapeo consume una cantidad significativa de tiempo, particularmente si se hace con el catéter de ablación (debido a que se toman puntos de manera individual).

\section{Recomendaciones}

\section{Clase I}

- Se recomienda el uso de tecnologías de mapeo tridimensional en la ablación de taquicardia ventricular isquémica con el fin de disminuir los tiempos de fluoroscopia (nivel de evidencia B).

- Se recomienda el uso de mapas de voltaje con el fin de delimitar zonas cicatriciales y ubicar los sitios con mayor potencial arritmogénico durante una estrategia de modificación de sustrato (nivel de evidencia $B$ ).

- Durante la ablación de LAVA, se recomienda el uso de técnicas de mapeo tridimensional con el fin de identificar y marcar las zonas de interés para facilitar la realización posterior de la ablación (nivel de evidencia B).

- Los sistemas de mapeo tridimensional son herramientas útiles para la detección y eliminación de canales en una estrategia de modificación de sustrato (nivel de evidencia B).

- Durante la ablación de sustrato mediante la ablación lineal, se recomienda la realización de mapas de activación con sistemas de mapeo tridimensional con el fin de demostrar bloqueo a través de la línea de ablación (nivel de evidencia C).

- Se recomienda el uso de sistemas de mapeo tridimensional con mapas de voltaje con el fin de identificar la zona cicatricial en caso de que se busque como desenlace la falta de captura dentro de la zona de cicatriz (nivel de evidencia C).

\section{Clase III}

- No se recomienda realizar ablación de taquicardia ventricular isquémica usando únicamente mapeo fluoroscópico (nivel de evidencia B).

\section{Bibliografía}

1. Principales causas de mortalidad en Colombia. Ministerio de Salud y Protección Social. [Acceso 4 Oct 2015]. Disponible en: https://www.minsalud.gov.co/salud/Paginas/Enfermedadescardiovasculares.aspx.

2. Colombia enfrenta epidemia de enfermedades cardiovasculares y diabetes. Ministerio de Salud y Protección Social; 2014 [Acceso 4 Oct 2015]. Disponible en: https://www.minsalud. gov.co/Paginas/Colombia-enfrenta-epidemia-de-enfermedades-cardiovasculares-y-diabetes.aspx.

3. Rodriguez T, Malvezzi M, Chatenoud L, Bosetti C, Levi F, Negri $\mathrm{E}$, et al. Trends in mortality from coronary heart and cerebrovascular diseases in the Americas: 1970-2000. Heart (British Cardiac Society). 2006;92:453-60.

4. Guía de práctica clínica para el Síndrome Coronario Agudo. Colombia. Bogotá: Ministerio de Salud y Protección Social Colciencias, Universidad de Antioquia; 2013.

5. Aliot EM, Stevenson WG, Almendral-Garrote JM, Bogun F, Calkins $\mathrm{CH}$, Delacretaz E, et al; European Heart Rhythm Associa- tion; European Society of Cardiology; Heart Rhythm Society. EHRA/HRS Expert Consensus on Catheter Ablation of Ventricular Arrhythmias: developed in a partnership with the European Heart Rhythm Association (EHRA), a Registered Branch of the European Society of Cardiology (ESC), and the Heart Rhythm Society (HRS); in collaboration with the American College of Cardiology (ACC) and the American Heart Association (AHA). Europace. 2009;11:771-817.

6. King JH, Huang CL, Fraser JA. Determinants of myocardial conduction velocity: implications for arrhythmogenesis. Front Physiol. 2013;4:154.

7. Nayyar S, Wilson L, Ganesan AN, Sullivan T, Kuklik P, Chapman $D$, et al. High-density mapping of ventricular scar: a comparison of ventricular tachycardia (VT) supporting channels with channels that do not support VT. Circ Arrhythm Electrophysiol. 2014;7:90-8.

8. Yokokawa M, Liu TY, Yoshida K, Scott C, Hero A, Good E, et al. Automated analysis of the 12-lead electrocardiogram to identify the exit site of postinfarction ventricular tachycardia. Heart Rhythm. 2012;9:330-4.

9. Josephson ME, Callans DJ. Using the twelve-lead electrocardiogram to localize the site of origin of ventricular tachycardia. Heart Rhythm. 2005;2:443-6.

10. Earley MJ, Showkathali R, Alzetani M, Kistler PM, Gupta D, Abrams DJ, et al. Radiofrequency ablation of arrhythmias guided by non-fluoroscopic catheter location: a prospective randomized trial. Eur Heart J. 2006;27:1223-9.

11. Strickberger SA, Knight BP, Michaud GF, Pelosi F, Morady F. Mapping and ablation of ventricular tachycardia guided by virtual electrograms using a noncontact, computerized mapping system. J Am Coll Cardiol. 2000;35:414-21.

12. Shurrab M, Laish-Farkash A, Lashevsky I, Morriello F, Singh SM, Schilling RJ, et al. Three-dimensional localization versus fluoroscopically only guided ablations: a meta-analysis. Scand Cardiovasc J. 2013;47:200-9.

13. Health Quality Ontario. Advanced electrophysiologic mapping systems: an evidence-based analysis. Ont Health Technol Assess Ser. 2006;6:1-101.

14. Khongphatthanayothin A, Kosar E, Nademanee K. Nonfluoroscopic three-dimensional mapping for arrhythmia ablation: tool or toy? JJ Cardiovasc Electrophysiol. 2000;11:239-43.

15. Andreu D, Berruezo A, Ortiz-Perez JT, Silva E, Mont L, Borras R, et al. Integration of 3D electroanatomic maps and magnetic resonance scar characterization into the navigation system to guide ventricular tachycardia ablation. Circ Arrhythmia Electrophysiol. 2011;4:674-83.

16. Mizuno H, Vergara P, Maccabelli G, Trevisi N, Eng SC, Brombin $\mathrm{C}$, et al. Contact force monitoring for cardiac mapping in patients with ventricular tachycardia. JJ Cardiovasc Electrophysiol. 2013;24:519-24.

17. Mountantonakis SE, Park RE, Frankel DS, Hutchinson MD, Dixit $\mathrm{S}$, Cooper J, et al. Relationship between voltage map "channels" and the location of critical isthmus sites in patients with post-infarction cardiomyopathy and ventricular tachycardia. J Am Coll Cardiol. 2013;61:2088-95.

18. Schilling RJ, Peters NS, Davies DW. Feasibility of a noncontact catheter for endocardial mapping of human ventricular tachycardia. Circulation. 1999;99:2543-52.

19. Rajappan K, Schilling RJ. Non-contact mapping in the treatment of ventricular tachycardia after myocardial infarction. J Interv Card Electrophysiol. 2007;19:9-18.

20. Pratola C, Baldo E, Toselli T, Notarstefano P, Artale P, Ferrari R. Contact versus noncontact mapping for ablation of ventricular tachycardia in patients with previous myocardial infarction. PACE. 2009;32:842-50.

21. Della Bella P, Pappalardo A, Riva S, Tondo C, Fassini G, Trevisi N. Non-contact mapping to guide catheter ablation of untolerated ventricular tachycardia. Eur Heart J. 2002;23:742-52. 
22. Marchlinski FE, Callans DJ, Gottlieb CD, Zado E. Linear ablation lesions for control of unmappable ventricular tachycardia in patients with ischemic and nonischemic cardiomyopathy. Circulation. 2000;101:1288-96.

23. Jais $P$, Maury $P$, Khairy $P$, Sacher F, Nault I, Komatsu $Y$, et al. Elimination of local abnormal ventricular activities: a new end point for substrate modification in patients with scar-related ventricular tachycardia. Circulation. 2012;125:2184-96.

24. Bogun F, Good E, Reich S, Elmouchi D, Igic P, Lemola K, et al. Isolated potentials during sinus rhythm and pace-mapping within scars as guides for ablation of post-infarction ventricular tachycardia. J Am Coll Cardiol. 2006;47:2013-9.

25. Komatsu Y, Daly M, Sacher F, Derval N, Pascale P, Roten L, et al. Electrophysiologic characterization of local abnormal ventricular activities in postinfarction ventricular tachycardia with respect to their anatomic location. Heart Rhythm. 2013; 10:1630-7.

26. Vassallo JA, Cassidy D, Simson MB, Buxton AE, Marchlinski FE, Josephson ME. Relation of late potentials to site of origin of ventricular tachycardia associated with coronary heart disease. Am J Cardiol. 1985;55:985-9.

27. Berruezo A, Fernandez-Armenta J, Andreu D, Penela D, Herczku C, Evertz R, et al. Scar dechanneling: new method for scar-related left ventricular tachycardia substrate ablation. Circ Arrhythmia Electrophysiol. 2015;8:326-36.
28. Santangeli P, Frankel DS, Marchlinski FE. End points for ablation of scar-related ventricular tachycardia. Circ Arrhythmia Electrophysiol. 2014;7:949-60.

29. Lin HT, Mann DE, Luck JC, Krafchek J, Magro SA, Sakun V, et al. Prospective comparison of right and left ventricular stimulation for induction of sustained ventricular tachycardia. Am J Cardiol. 1987; 59:559-63.

30. Robertson JF, Cain ME, Horowitz LN, Spielman SR, Greenspan $\mathrm{AM}$, Waxman $\mathrm{HL}$, et al. Anatomic and electrophysiologic correlates of ventricular tachycardia requiring left ventricular stimulation. Am J Cardiol. 1981;48:263-8.

31. Nayak HM, Verdino RJ, Russo AM, Gerstenfeld EP, Hsia HH, Lin $D$, et al. Ventricular tachycardia storm after initiation of biventricular pacing: incidence, clinical characteristics, management, and outcome. J Cardiovasc Electrophysiol. 2008; 19:708-15.

32. Vergara P, Trevisi N, Ricco A, Petracca F, Baratto F, Cireddu M, et al. Late potentials abolition as an additional technique for reduction of arrhythmia recurrence in scar related ventricular tachycardia ablation. J Cardiovasc Electrophysiol. 2012;23:621-7.

33. Di Biase L, Santangeli P, Burkhardt DJ, Bai R, Mohanty P, Carbucicchio $C$, et al. Endo-epicardial homogenization of the scar versus limited substrate ablation for the treatment of electrical storms in patients with ischemic cardiomyopathy. J Am Coll Cardiol. 2012;60:132-41. 